\title{
Health Inequity by Design: Waiting Rooms and Patient Stress
}

\author{
Matthew D. Lamb* \\ Department of Communication Arts and Sciences, The Pennsylvania State University (PSU), University Park, PA, United States
}

Little attention has been paid to health inequities designed into the physical spaces themselves. Clearly design is an important part of patient care. Design is simultaneously a complex system itself while existing as part of a larger complex (healthcare) system. For example, it is not enough to say that a patient experiences more stress because she/he is being treated in a hospital in a lower income area. The key, here, is that evidence demonstrates design as an important component, systemically, in healthcare. We know this to be true and base re-design efforts on this fact, but only in certain places. The central addition of this study is to point out that hospitals in higher income areas utilize the waiting room's ecology and its influence on patient stress and care. Efforts to intervene,

OPEN ACCESS

Edited by:

John Parrish-Sprowl,

Indiana University, Purdue University Indianapolis, United States

Reviewed by:

Rod Carveth,

Morgan State University, United States

Pamara F. Chang,

University of Cincinnati, United States

Linda Ziberi,

Rochester Institute of Technology Kosovo, Prishtinë, Kosovo

*Correspondence: Matthew D. Lamb mdl20@psu.edu

Specialty section: This article was submitted to Health Communication, a section of the journal Frontiers in Communication

Received: 12 February 2021

Accepted: 27 August 2021 Published: 10 September 2021

Citation: Lamb MD (2021) Health Inequity by Design: Waiting Rooms and Patient Stress.

Front. Commun. 6:667381. doi: 10.3389/fcomm.2021.667381 through design, in waiting room ecology have consequences to equitable access to healthcare. Therefore, this study examines the implications of health inequities designedinto physical space. Additionally, this study seeks to forefront the influence communication ecologies have in addressing health inequities. Innovations in addressing mental health needs in humanitarian settings: A complexity informed Action Research Case Study. Frontiers in Communication: Health Communication. 10.3389/fcomm.2020.601792 para 19, 2020). Thus, the purpose of this paper is to investigate, but also articulate, the ways design decisions impact people unequally and perpetuate health inequalities. To do so, this study investigates the communication ecologies of waiting rooms and their influence on patient stress and health equity and elucidates under-examined systemic components patient stress and well-being.

Keywords: waiting rooms, communication complex, communication ecology, patient stress, health inequities

\section{INTRODUCTION}

Interior design has made its way into the consciousness of the healthcare landscape. Healthcare Interior Design, or HCID, has played an increasing role in hospital waiting rooms. Stress can make us sick and further complicate existing conditions (Sternberg, 2010). The importance of space in wellbeing and healing is also well-established (Sternberg, 2010). Put plainly, the organization of waiting room spaces can stress us out. They also hold the potential to calm us, help release our stress, and aid in healing. Spatial elements which influence patient stress can be crowded or busy spaces, noise, light (too much, too little), foul odors, and spaces which are confusing or difficult to navigate (Sternberg, 2010). Unfortunately, many of our memories from experiences in hospitals would most likely associate hospital waiting with rooms such characteristics. How often have you associated hospital waiting rooms with a sense of calm or relaxation? The organization of space and the impact of design (intentional or not) can be felt, literally.

Parsley Health, a start up in the health industry, is changing the way healthcare feels and they are starting with their waiting rooms. Their model embraces the concept of social determinants of health 


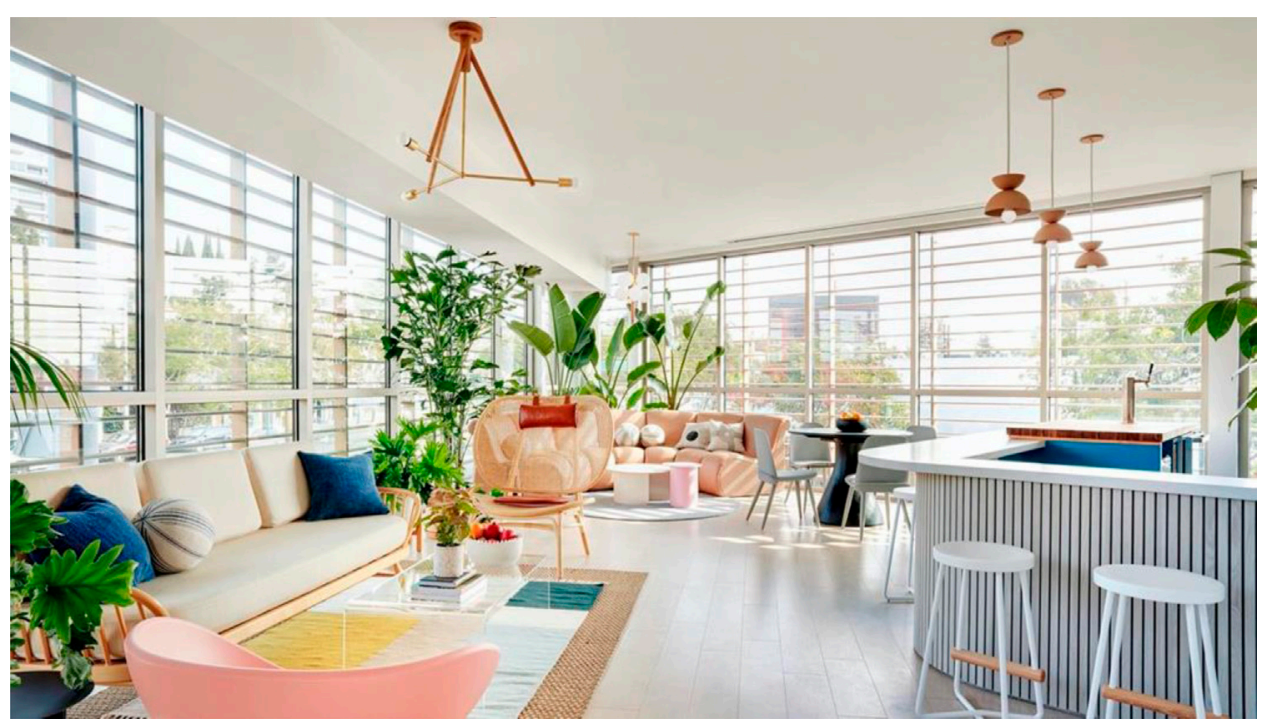

FIGURE 1 | Lounge: Credit Kara Ladd, Architectural Digest (2019), photography by Trevor Tondro

$(\mathrm{SDoH})$ and is built around finding better ways to treat and engage the whole person, holistically and collaboratively. They base their approach on research (most often, Braverman and Gottlieb, 2014; Hood et al., 2014) which makes the argument that nearly $90 \%$ of our health is determined by what we eat, how we move, our environment, and how we manage stress. As a result, Parsley's waiting rooms, in fact their entire office space, is designed around the concept of biophilia. This design philosophy uses natural elements to improve the environment for the physical and mental wellbeing of the space's user. The purpose of this approach is to ease patients' anxiety, mitigate stress, and contribute to patients' overall health through purposeful design.

Biophilic design is especially notable in the waiting lounge (Figure 1). The waiting rooms of a Parsley Health location come complete with a stress-reducing array for the senses. Live plants are visible and perfectly positioned in various corners of the room. These give a feel for nature and freshen the air. Essential oil blends are used to engage the olfactory senses. Scents such as lemon, basil, and Cedarwood Virginian are used. These oil blends, custom-made for Parsley Health, possess mild antiseptic properties and are meant to promote immunity, and most notably, a feeling of calm. Waiting rooms are also designed with meditation nooks which not only provide a space for privacy but also help patients connect with their breathing, a technique which increases awareness, intention, and lowers stress.

Floor-to-ceiling windows provide access to natural light in the purposely large open spaces of the lounge. Compact Fluorescent Lighting (or CFL) has been eschewed in most biophilic-centered designs, but especially at Parsley Health. CFLs have been shown to have a slight flicker which has been linked to irritation of our nervous systems and can lead to headaches (Dunckley, 2014). Wood is also used in abundance, e.g., for floors, chairs, shelving, etc. Natural materials help us feel connected to the natural world.
For example, Baldwin (2012) showed the use of plants and natural greenery in waiting rooms helped to reduce patient stress. The intent is to establish an emerging trend in the practice of healthcare and its subsequent research; design is a key factor which contributes to patients' overall health.

\section{Purpose Justification}

I open the study with this example because complexity, and, as will be further focused, communication ecologies, often are felt with more effect intuitively rather than cognitively. We have all had experiences in which the health care milieu was stressful. From crowded waiting rooms, to crying babies, to the person coughing next to you, most of us has a story to tell about waiting room stress.

The World Health Organization (WHO) defines health equity as the "absence of avoidable, unfair, or remediable differences among groups of people, whether those groups are defined socially, economically, demographically or geographically or by other means of stratification" (World Health Organization WHO, 2020). Their definition continues to define equity in health as every person having a fair opportunity to attain "their full health potential," and perhaps most importantly for our purposes here, that no one "should be disadvantaged from achieving this potential" (World Health Organization WHO, 2020). Thus, an overlooked element in healthcare, specifically in healthcare design, is that by adding stress to a person already dealing with the stress of health challenges would play a significant role in the process of a patient's full health potential.

Little attention in healthcare has been paid to health inequities designed into the physical spaces themselves. Design is an important part of patient care. It is simultaneously a complex system itself, while existing as part of a larger complex (healthcare) system. For example, it is not enough to say that a patient experiences more stress because she/he is being treated in a hospital in a lower income area. The key here is that evidence 
demonstrates design as an important component, systemically, in healthcare. Thus, some re-design efforts are based on this evidence, but only in certain places. The central addition of this study is to point out that hospitals in higher income areas intentionally utilize the waiting room's ecology and its influence on patient stress and care. Efforts to intervene, through design, in waiting room ecology have consequences to equitable access to healthcare.

This study examines the implications of health inequalities designed-into physical space. Additionally, it seeks to forefront "the interplay between communication and health, as well as how health is shaped and maintained" (Parrish-Sprowl et al., 2020 para 20). Thus, the purpose of this paper is to investigate, but also articulate, the ways design decisions impact people unequally and perpetuate health inequalities. To do so, the study investigates the communication ecologies of waiting rooms and their influence on patient stress and health equity and elucidates under-examined systemic components of health.

\section{Defining a Complex System}

Cohn et al. (2013) provide a useful definition of complexity. They describe complexity as "a dynamic and constantly emerging set of processes and objects that not only interact with each other but come to be defined by those interactions" (p. 40). Complexity theory is a post-Newtonian paradigm which challenges more traditional, linear, cause-effect perspectives. Newtonian, or linear systems, perspectives have traditionally approached public health matters being informed by methods based on linear models of cause and effect (Rutter, 2018). The goal has been to identify a cause, implement a solution to that cause, and evaluate its effectiveness over time. Linear, or cause and effect approaches, to public health challenges can be reductive and decontextualized, thus lacking the depth of the overall, more complex picture needed for effective responses.

Complexity-based approaches characterize how systems behave. They give us a prospective to investigate the multiple ways system components/elements interact. Complexity-based approaches provide a timely, practical, and useful framework to an environment-centered framing of public health. They provide a framework to see human health as contextual and environmentcentered, consisting of the people, their activities, wants, needs, values, lifestyles and other aspects of culture. Reframing the environment in this way affords a deeper, more nuanced approach to human health.

\section{Communication Complex (CC)}

CC shares complexity approaches' move away from Newtonian driven insights into human experience. Parrish-Sprowl and Parrish-Sprowl (2014), point out that such approaches are not wrong but simply no up to the task of engaging human experience with the robustness it requires. According to the authors, CC stands in contrast to a "foundationalist approach to human experience," which "essentializes and reifies phenomena by treating them as "found" things "out there"” (Parrish-Sprowl and Parrish-Sprowl, 2014, p. 6). Parrish-Sprowl et al. argue for a more "quantum understanding" of communication phenomena. They describe this shift in thinking, as a necessary expansion on "foundationalist epistemological and methodological assumptions of a traditional social science" (2014, p. 5). Similar to other complexity approaches, CC challenges traditional, linear understandings which fail to fully capture the systemic nature of human communication.

This shift in theoretical perspective allows us to more effectively analyze the interactions between space, design, patient, health care and their role in patients' whole health. For example, with more traditional approaches, patient stress might be caused by poor lighting. Research illuminates that fluorescent lighting can lead to headaches. Change the lighting, change the stress. Problem solved. The lighting was "found" to be a cause and thus a solution was implemented. Yet, CC encourages us to take a closer look. This perspective allows us to view the lighting as part of an interplay between patients and their whole health experience. The stress inadvertently caused by poor lighting can then lead to challenges in healthcare because that stress has made it difficult to consume information and make decisions. Thus, CC helps us frame the entire system. For example, decisions on lighting, cost, and maintenance function as components of the system just as much as patient stress, health literacy, and even the doctors' mental health providing the care.

CC offers a quantum consideration to the study of human communication. Its more dynamic approach considers the everchanging, ever-adapting process of nebulous interactions (between people, space, past experiences, and many more) which constitute meaning. As Parrish-Sprowl et al. (2014) write, a more quantum view of human functioning "sees movement within the embodied self" and "between embodied selves" as a flow of energy and information in nonstop, simultaneous, dynamic process that operates like quarks, defying the rules of logic and connecting people across time and space" (p. 6). The CC perspective forefronts that we live in communication with others rather than the more common simplistic view of communication as just a means of transmitting information between individuals, thus reifying the appearance of separation consistent with a Newtonian understanding of the world (Parrish-Sprowl, 2013, 2014). The advantage for applying the CC framework to waiting rooms is in its ability to analyze the nebulous ecology in which healthcare is performed.

\section{METHODS}

Evaluation of communication ecology necessitates a complex approach. The analysis which follows includes a collage of images, and personal experiences using various information and communication technologies (ICTs). Images were collected by committee, i.e. images were taken and submitted by volunteers who visited various hospital waiting rooms and areas. The personal experiences included for analysis were the author's own visitations to hospital sites.

The chosen research method for this investigation draws from Ahlin and Fangfang (2019) conception of "field events" The critique of the waiting spaces involved here fully accepts the call for complexity as it is grounded in cultural theory, 
architectural critique as well as neuroscience. The term "multisited fieldwork" was coined to encompass studying several geographic sites which could be added together. The researcher may not be able to visit all the locations in person (Marcus, 1995). Multisited fieldwork encompasses "different, complexly, connected real-world sites of investigation" (Marcus, 1995, p. 102) or "various social spheres of everyday life, legal institutions, mass media, or policy discourse." (Ahlin and Fangfang, 2019, p. 3).

Ahlin and Fangfang (2019) update the multisited method to include the use of modern technologies such as mobile phones, laptop computers, software such as Zoom, FaceTime, Facebook Rooms, etc. Their conceptualization of multisited fieldwork offers a useful method known as "field sites" (Ahlin and Fangfang, 2019). They include virtual, or digital fieldwork with the use of ICTs such as video conferencing or sharing videos and images with and among study participants. They argue the field event shifts the focus from the field as something "situated in geographic and social spaces ("site"), whether physical or digital/virtual ... towards understanding the field as a collection of "events" that are co-created within specific practices by ethnographers, their study participants, and ICTs" (Ahlin and Fangfang, 2019 p. 4). Multiple participants were asked to submit images of their visitations to the hospital. Thus, each image is a unique perspective from each participant. I as the researcher am learning along with each volunteer about what they see, how they interpret the space, as well as co-creating the ethnographic practices underlining this research.

This study took place during the COVID-19 pandemic. Therefore, fieldwork presented unique challenges. These challenges were compounded given the focus of the study, hospitals waiting rooms. However, encountering these challenges also adds a useful dimension to the study. Practices such as social distaining, different practices in waiting lines, placing a flag on a waiting room seat to denote the seat is in need of cleaning, all complicated the communication ecology but also gave further insight into the communication ecology of the spaces studied.

\section{DISCUSSION}

The analysis which follows is informed by the framework ParrishSprowl and Parrish Sprowl (2017) have termed Communication Ecology. Consistent with the framework of CC, communication ecology allows us to go deeper into the communicative robustness of environments under study. As described by Parrish-Sprowl et al. (2017), communication ecology is a "web of meaningmaking" (2017, para 2). This "web" "shapes our thoughts, physical sensations, feelings and actions and at the same time, our thoughts, physical sensations, feelings and actions contribute to the system itself" (2017, para 2). Communication ecology conceptualizes how we are "immersed in messages and meanings all around us, from environmental cues in our surroundings, to things we say and do, both face-to-face and in the media" (2017, para 2). Additionally, Parrish-Sprowl and Parrish-Sprowl, 2017 point out, we are able to learn about

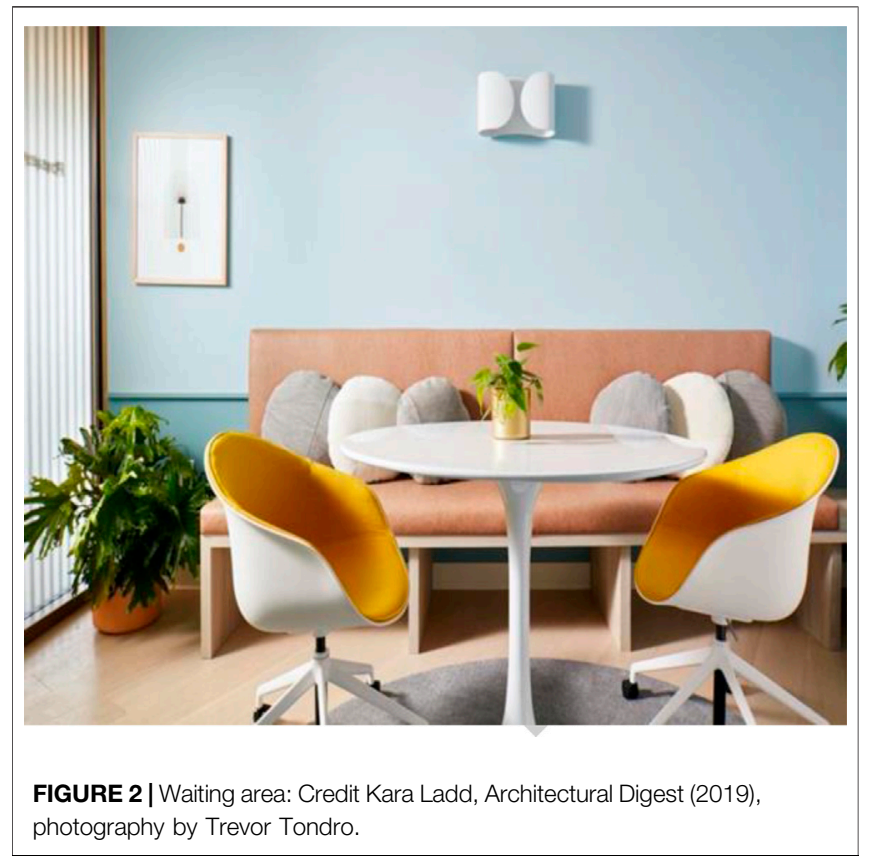

individuals' health and well-being by exploring the "interrelationships between people and their environment, as well as with each other" (para 2).

Communication ecology is similar to the framework of media ecology. As Neil Postman (2000) described, the Petri dish served as a biological metaphor for understanding how a substance grows in a culture. Postman substituted the terms substance and technology describing the modified metaphor as the fundamental principle of media ecology. As he states, a "medium is a technology within which a culture grows" (p. 10). Put plainly, technology gives "form to a culture's politics, social organization, and habitual ways of thinking" (Postman, 2000, p. 10). The term ecology is used to frame the interconnection among elements in our environment. As media is the "substance" in which a culture grows, interactions amongst media elements are studied for the ways in which those interactions "between media and human beings give a culture its character and ... help a culture to maintain symbolic balance" (Postman, 2000, p. 11). Media ecologists concern themselves with the multitude of interactions, and potential for interactions, between elements and how those interactions constitute what gets called culture.

To see how the communication ecology framework helps us investigate potential health inequalities linked to the design of physical space, we turn to an example of different approaches to flex rooms in waiting areas.

\section{Flex Rooms}

For the most part, a flex room is used as a sort of transitional space. Healthcare providers may reorient or redecorate the space for different uses as need requires. Patients may be transitioning from the waiting area to another care-oriented space, e.g. going from the waiting room to the exam room. The flex room allows patients to fill out additional forms such as symptoms 


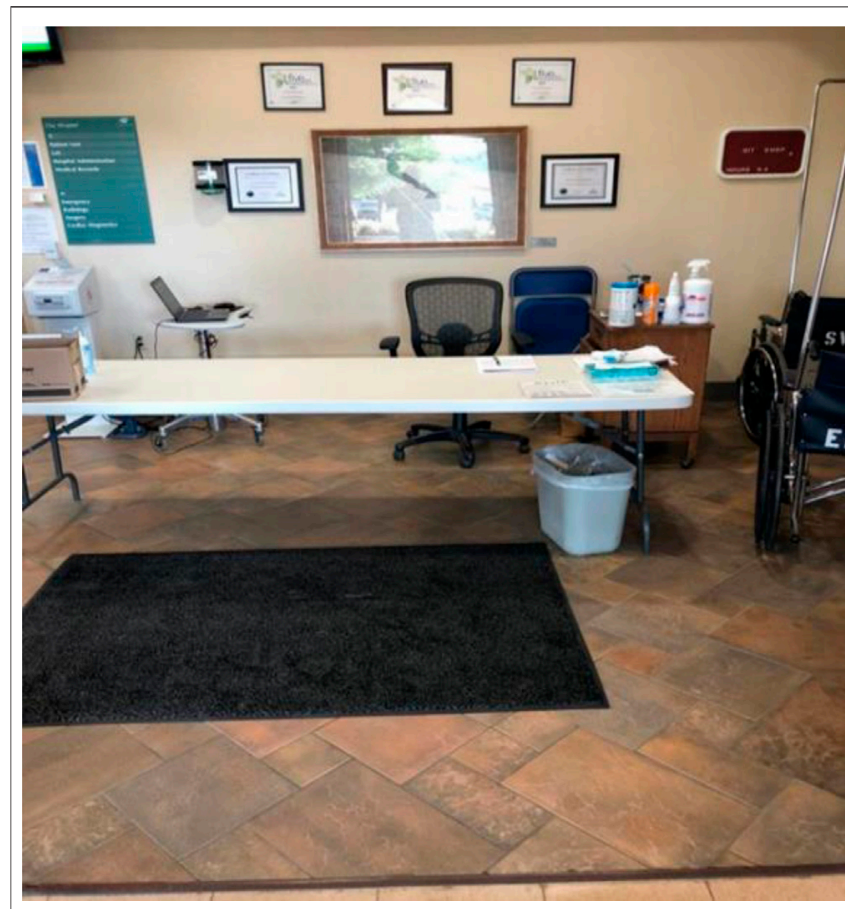

FIGURE 3 |Waiting area: Hospital in Carmel, Indiana (2019), photograph by Lisann Good-Burton.

questionnaires or consult with health care workers. In many cases this becomes the secondary waiting area.

The two examples demonstrate vastly different spaces. The first (Figure 2) is designed and used by Parsley Health in New York City. The second (Figure 3) is a flex space used in a hospital in Carmel, Indiana. As you view the pictures, consider the feeling of the space. Consider how each element in the space communicates. Think about your relation to each element in the space. How does it make you feel, what does it make you think, how does it communicate to you? Do you feel like a coauthor of your own care? Do you feel like a medical object? You may not consciously identify any of these connections with the space. However, the key to understanding communication ecology is to first accept that these spaces and the relationships we have with and to them are not neutral. As Hilary Koyfamn, Parsley Health's interior designer, says "We paid close attention to the color palette to instill a sense of calm, activating a parasympathetic state, otherwise known as rest-and-relaxation" (cited in Ladd, 2019).

The flex room by Parsley taps into our sense of equality with the caregiver. The use of a round table to mitigate perceptions of power hierarchy and encourage feelings of inclusion. The chairs with softer, rounded edges are chosen for comfort and encourage relaxation. The same consideration is given to the couch and the use of blue painted walls. The room is filled with natural light and greenery. All of these elements help produce an ecology that invites activation of the parasympathetic nervous system. The room invites patient and provider to engage with one another in a more parasympathetic receptive state versus a more stressinduced reactivate state. The medical object is made more complex. The whole person becomes qualitative instead of quantitative.

The flex room in the second image is more traditional and function-oriented in its arrangement. Stated another way, the room's arrangement is differently functioned, i.e. the flex space here is attuned to the medical object of the symptom or disease. It does not encourage a feeling of collaboration or communicate attention to the whole health of the individual. With elements such as the plastic card table or mobile laptop stand the space communicates a power structure and a sense of distance between the patient, or medical object, and the healthcare worker.

While care is accessed and provided, both variations on the theme demonstrate a stark contrast in communication ecology. Applied to examples of haute design, communication ecology shows that the space is actively, purposefully manipulated to evoke an intended feeling in the patient, one more in line with well-being, healing, and lower stress. The room, in this sense, is similar to the ways in which we carefully arrange our words in a persuasive message. We strategically arrange the words in the message to have an intended effect. Haute design works much the same way. Waiting rooms are designed through the arrangement of myriad factors such as lighting and access to sunlight, natural plants, soothing soundscapes, fresh vs recycled air, artwork, as well as the legibility of the space (how easy it is to navigate) and tell where you're going and what you're supposed to do). Such design elements offer an overall modern waiting space and pathway to care which looks, feels, sounds, and even smells the part. Attention paid to physical space is directly related to the evidenced importance for the ease of stress and patient comfort is to aide the patient in developing a sense of calm and security.

The pictures demonstrate the different ecologies of each space. The different ecologies of space are simply different approaches; each is capable of treating patients and providing care. The point being, is that health equity concerns itself with the whole person and the absence of avoidable, unfair, or remediable differences among groups of people. This includes spaces of care as well. Because we are not separate from our environments nor context, message success is not enough to say health care or healthy communication has occurred. We do disservice to health equity when we consider the message only when it is but one part of a system hereto referred to as the communication ecology.

Yet, even the best interior design cannot guarantee improved health or even more effective communication. However, the evidence demonstrates that these elements matter. Built spaces have an impact on the ways in which people interact. Better conversations with your doctor can indeed begin with better waiting areas. For example, research conducted by Ayling et al. (2017), showed that patients with a positive frame of mind actually improved vaccine effectiveness. Not every patient in the waiting room is there for a vaccine. Still, when we see the waiting area not merely as physical space but as an ecology, a web of meaning-making, we can then take seriously that such spaces do in fact influence and shape our thoughts, physical sensations, feelings and actions, knowing too that those also contribute to the ecology itself and care of the patient. This example and 
framework prime our thinking as we engage further examples to expand our notions of health equity.

We now have a groundwork and framework to see how health inequities may not be a simple matter of access or lack of motivation to care for oneself. CC gives us the ability to analyze how health inequities can be designed into healthcare facilities even when the boxes of access and motivation or myriad other traditionally agreed upon determinants are checked.

\section{The Bioactive Nature of Communication Ecologies}

When considering efficiency in seeing patients but also in constructing spaces for care, large numbers of people are being ushered into quickly built, affordable, stick-over-podium type clinics. Stick-over-podium construction allows structures to be built quickly and efficiently. The advantages are many when it comes to getting clinics built and operational. Getting people in and out as efficiently as possible ensures a large group of an under-serviced or low resourced community will be seen by medical professionals and be treated with appropriate medicine. Yet, the large volume also means that care for the majority of people will be quantitative and not qualitative, i.e. seeing as many patients as possible acts as the prime directive over personal, collaborative, whole health approaches.

Here waiting rooms are studied as communication ecologies. Everything from the physical set up of the room, the lighting, sounds, materials, people, etc. Each contributes to the waiting room as a web of meaning-making because each person from her/ his own vantage point is immersed in the messages and meaning all around them. The human brain is a social brain (Cozolino, 2014). Thus, every interaction is a meaning making process which occurs continuously in each person. It is paramount to remember that perception is an actively formed opinion of the world rather than a passive reaction to sensory input from it (Ramachandran, 2011). Every stimulus which impinges on an organism has an effect on it physiologically. The waiting room can cause us stress, anxiety, and even sheer anger, none of which is advantageous for creating healthy, long-term communication and health care for patients and healthcare workers alike. In a very real sense, the space itself communicates just as much as the interpersonal messages we spend most of our time studying. The space of the waiting room is a communication ecology in as much as it helps to shape our thoughts, physical sensations, feelings, and actions.

As the example demonstrates, the complex nature of the spatial communication ecology renders traditional linear, cause and effect approaches ineffective as frameworks to study the link between space and stress, and articulating their relationship to healthcare inequalities. Communication ecology as a framework is especially useful in this context. The aforementioned spatial stressors are certainly not an exhaustive list nor would you be able to separate them as variables. Noise for example may come in and out of the person's conscious awareness but is constantly active in the environment. Perceptions of crowded spaces and foul odors will wax and wane with conscious attention. Although not on our minds right now, the cumulative effects of each of these factors

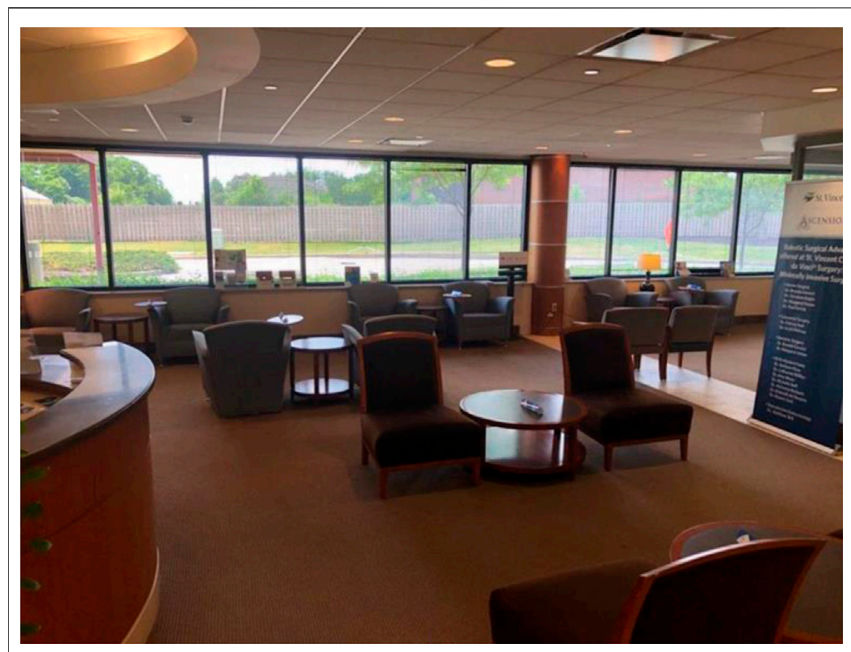

FIGURE 4 | Waiting area: Hospital in Carmel, Indiana (2019), photograph by Lisann Good-Burton.

again are not neutral but have influence in the overall communication milieu, or ecology, of the space.

The subsequent sections analyze four components of a waiting rooms' communication ecology. These are not meant to be a model of the ecology or a definitive list. These four components of the complex system simply are used to demonstrate how the environment itself can trigger stress responses in patients. They are also used to provide real-world examples of how the choices in design, organization, maintenance, flow and use patterns have systemic effects which, if left uncriticized and evaluated, can drive health inequities for those who depend on those spaces for health care.

\section{Organization of Space and Stress}

The way in which physical space is organized has been shown to influence use and movement which become routine (Lamb, 2014; Lamb, 2014b; Lamb, 2017). Airports, for example, were early adopters with concern for the influence physical space has on us. The design prompts people to wait in a single file line and then go to the next available security point. This is predicated upon reducing anxiety and stress. Hullinger (2020) reported that a single queue actually lowers stress levels because people perceived it with a sense of fairness. When people see another line moving faster they can experience anxiety, stress, and deeply rooted senses of resource protection. Further studies have shown the environment we occupy has a significant influence on how our brain functions. For example, neuroscientists have shown that in a natural environment, our brain activities are more disentangled and meditative (Azzazy et al., 2020). Yet, when we are in a human built environment, the brain has shown higher levels of stress (Azzazy et al., 2020). The following sections highlight several facets of the built environment's relationship to us and our experiences of stress in waiting room settings.

The image below (Figure 4) is a typical waiting room in the US. There is natural lighting (which will be discussed later in the paper). There is plenty of seating and the space itself is reasonably easy to navigate. 


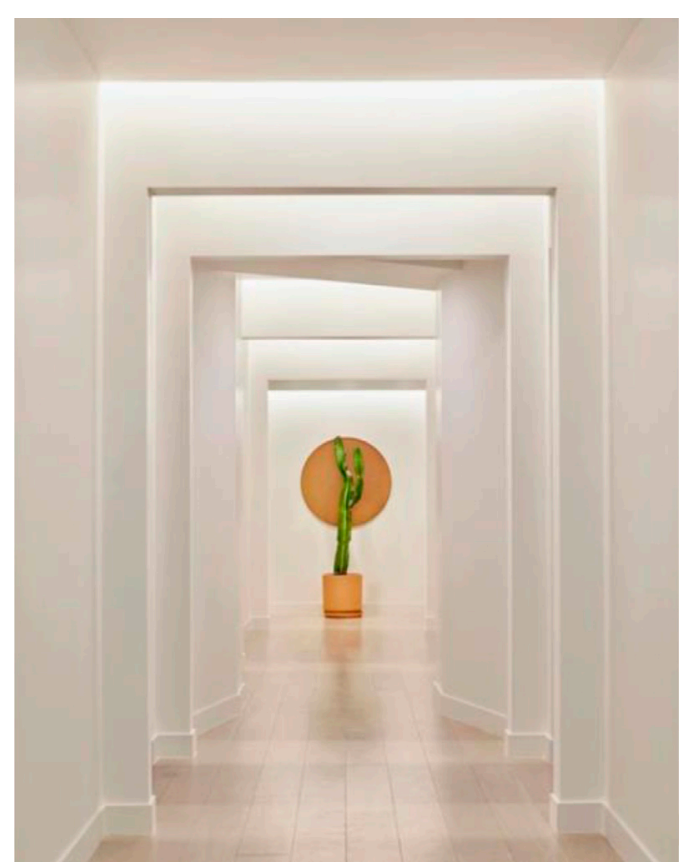

FIGURE 5 | Zen-like hallway: Credit Kara Ladd, Architectural Digest (2019), photography by Trevor Tondro.

One component of this room is the direction of eye gaze. This is not to say that people shouldn't make eye contact. Eye contact can trigger stress responses. Eye contact as part of the communication ecology emerges from the interactions between people and between people and space. Direction of eye gaze aids in our prediction of how others are likely to behave (Cozolino, 2014, p. 174). This becomes even more important when we dig deeper into the perception process. The amygdala is an appraisal organ. It helps us make positive associations with aspects of the environment to guide and control approach-avoidance behavior (Cozolino, 2014). When the space makes eye contact challenging, or difficult, or the waiting area is confusing, frustrating, etc. it has real effects on our appraisals of the environment, our place within it, and our stress responses as a result. Our "amygdala connects present experiences with appraisals from out past" (Cozolino, 2014, p. 175). So, immediately, we may connect the waiting room to negativity simply because of past experiences. These spaces have not really changed that much over the decades. The amygdala becomes active in responses to interpersonal threat, for example, when we are being looked at by others, and when we see others express stressful or fearful facial expressions (Cozolino, p. 178).

\section{Wayfinding}

In addition to adding higher levels of stress, buildings themselves can make us sick. There is even a term for this, "sick building syndrome" (Molhave, 1989). Spaces which are confusing or difficult to navigate and crowded or busy spaces, can affect our ability to feel comfortable and relaxed. Achieving our

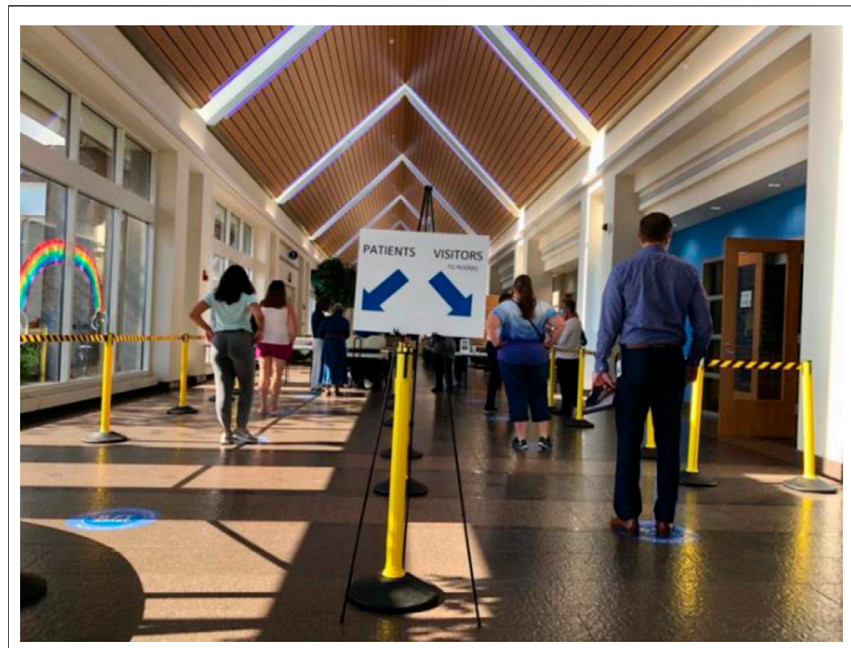

FIGURE 6 | Waiting line: Hospital in Carmel, Indiana (2019), photograph by Lisann Good-Burton.
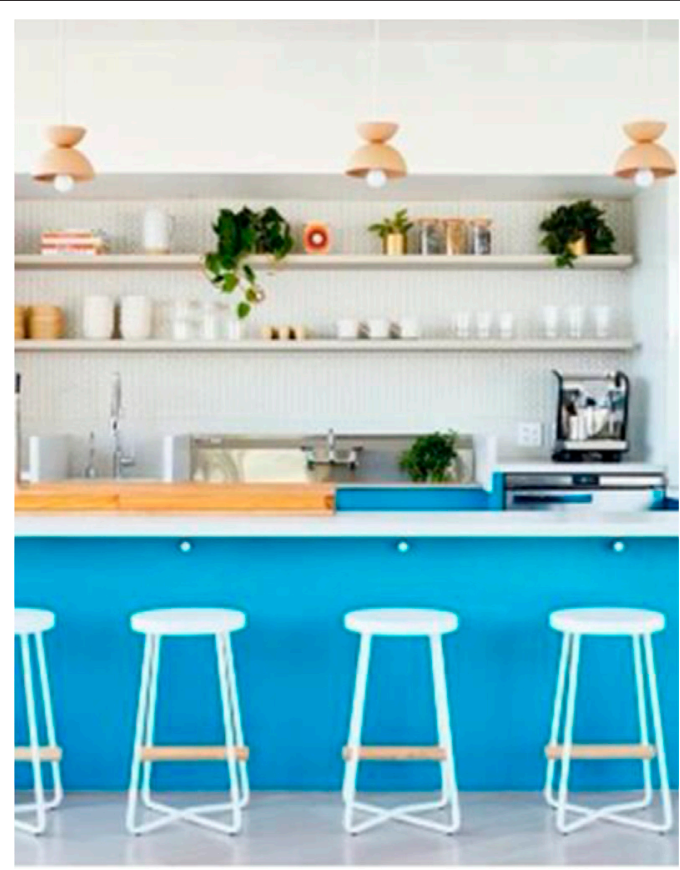

FIGURE 7 | Cafe: Credit Kara Ladd, Architectural Digest (2019), photography by Trevor Tondro.

goals effortlessly with navigation is vitally important. Wayfinding is how we make sense of and navigate physical space. Brunye et al. (2010) describe wayfinding as knowing of an existing place and knowing how to reach a desired goal as quickly and effortlessly as possible. The interaction is mediated through our emotional reactions to the space. People's experience of the properties of the space affects their mental condition (Coburn et al., 2017). For example, research has shown that too much automation and control has been shown to shown to 


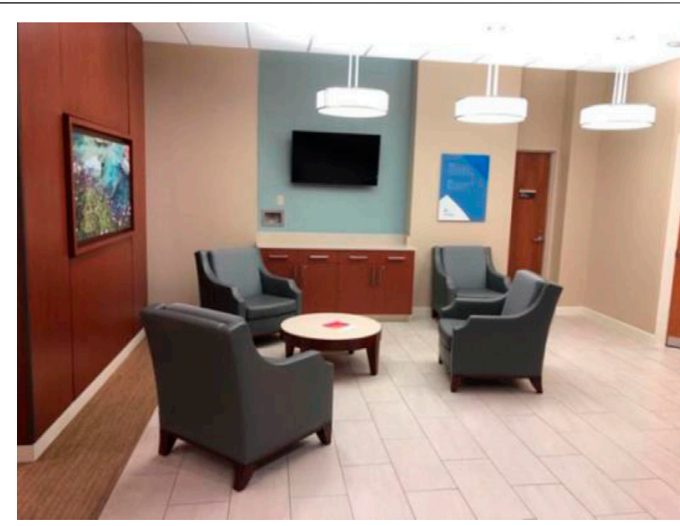

FIGURE 8 | Waiting area: Hospital in Carmel, Indiana (2019), photograph by Lisann Good-Burton.

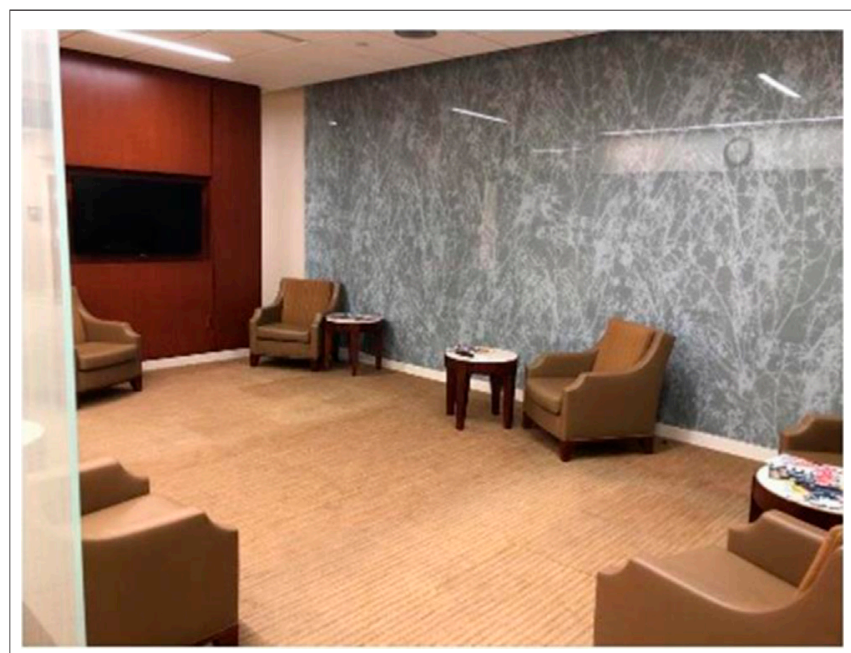

FIGURE 9| Waiting area: Hospital in Carmel, Indiana (2019), photograph by Lisann Good-Burton.

reduce people's feelings of comfort because it reduced their feelings of control (Tamas et al., 2020). There is a delicate balance between spatial makeup and feelings of stress. Most individuals desire a certain amount of autonomy in their use of space.

Consider the differences between Parsley Health's approach (Figure 5) and a hospital in Indianapolis, IN (Figure 6). The immediate differences are due to COVID-19. The Indianapolis hospital has had to incorporate new wayfinding procedures. The lines are clearly marked so stress triggers have been addressed. The Parsley Health approach to wayfinding is more organic. The hallway in (Figure 7) is designed to emit rays of sunshine leading you down a hallway. Each layer is meant to provide the patient with an experience of nature and feelings of calm. While it is hard to believe a patient would confuse the hallway with a walk in nature, perhaps it is the thought that counts. Meaning, most will understand these as design elements but seeing a different approach means feeling a different sensation. When you are aware the space is trying to interact with you in positive ways, you are perhaps more likely to oblige.

These two waiting rooms create a perceptual difference. Even without being in the space themselves.

First, Figure 8 is the waiting room reimagined by Parsely Health as a waiting lounge. Notice the natural light, attention to natural materials, as well as the use of natural elements such as plants and views of nature. Figure 9 was taken at a hospital in Richmond, Indiana. Functionality is the key here with little to no design attention paid with regard to patient stress responses. Revisiting the notion of what is being created here, we can see both approaches construct a message rhetorically with the symbolism of the space.

One constructs an experience which invites the patient to relax, feel welcome, and safe. The other, while not overtly trying to trigger stress, does little to mitigate such a response. Again, these examples are not to praise or condemn each space. The point of these examples is to show that space matters. It is not neutral and is an often-overlooked component in creating the types of communication ecologies in healthcare facilities that lead to health equity and ensure every person has a fair opportunity to attain their full health potential.

\section{Odors}

As previously noted, the waiting rooms used for this research were studied during the COVID-19 pandemic. Smells during the pandemic are different than prior to the outbreak. For example, each waiting room had cleaning teams which used generous amounts of cleaner and/or sanitizer. One cannot help but wonder if the odors of sanitizer was somewhat deliberate to communicate "clean." What is important about odors in the waiting room is that pheromones are believed to be the earliest form of social communication (Cozolino, p. 95). The vomeronasal system is specialized to detect "social smells" and consists of the vomeronasal organ, the vomeronasal nerve, and the accessory olfactory bulb. Questions still remain regarding the importance of pheromonal communication. For example, evidence shows the brain's developed neocortex enables people to process multiple channels of sensory information simultaneously, thus rendering reliance on pheromonal communication increasingly less important (Cozolino, p. 95). However, Grosser et al. (2000) point out that pheromonal communication plays a significant role in the ways people influence each other's moods.

As mentioned, the waiting rooms' odors were predominantly that of sanitizer. However, many other smells were present. For example, in the Richmond, Indiana hospital, body odor was noticeable in the waiting room. Endevelt-Shapria et al. (2017) demonstrated that people detect cues from body odors. An interesting component of body odor is that its interpretation is largely cultural and can be used to make judgments about class (Hannigan, 1995). This odor in particular can bring about feelings of anxiety and stress. Patients may interpret class and associate that with the sense of a different set of cultural and personal values. Patients may also sense feelings of fear and empathy. 
In fact, olfaction has been found to have a close relationship with the limbic system and emotion. Spinella (2009) found a correlation between smell identification and people feeling a sense of empathy. These cues have autonomic responses which can affect behavior through the subliminal detection of chemosensory cues, for example, through the body odor of someone experiencing fear (Endevelt-Shapria et al., 2017). The autonomic nervous system is a control system that acts (mostly) unconsciously and regulates functions such as heart rate, digestion, respiratory rate, and pupillary response, amongst several others. The autonomic nervous system is the primary mechanism regulating fight-or-flight responses.

This is significant when understood through the lens of a communication ecology. Human odors convey information. In short, odors communicate. For example, sweat contains many potential odorants which convey emotional information in humans (Endevelt-Shapria et al., 2017). This is not confined to human odors. We detect and react to all manner of odors. The interesting component is that odor, unlike other sensory systems, does not pass through the thalamus. Smells are routed to the cortex. Thus, odor information is transmitted directly to the limbic system, which typically is associated with memory and emotional processes. "This provides olfaction with a unique and potent power to influence mood, acquisition of new information, and use of information in many different contexts including social interactions" (Sullivan et al., 2015).

Olfaction impacts our orbitofrontal cortex, mediodorsal thalamus, and the amygdala (Endevelt-Shapria et al., 2017). Put plainly, odors can affect your ability to interpret information, interact with people, and make decisions. Simply by taking a closer examination of the communication ecology, we find another component of the meaning-making system. While odors may not be at the forefront of our consciousness at all times, they are nonetheless there at all times. Odors are communication messages whose influence is not neutral. They impact how we acquire new information and our social interactions. They are yet one more piece of the waiting room's ecology that influences our emotions and how we take in new information. Consider the impact this knowledge has, for example, for a patient and her/his health literacy or how she/he makes decisions about care for themselves or a loved one.

\section{Sound/Noise}

Noise plays a significant role in patients' perceptions of the hospital's environment. Hospital noise has been proven to produce undesired physiological and psychological responses in individuals which impact mental and physical health (Cunha and Silva, 2015). Noise has a direct impact on patient wellbeing (Cunha and Silva, 2015) and healing (Sternberg, 2010). Noise is listed among the most stressful and distracting nonclinically relevant events (Akansel and Kaymakci, 2008; Mazer, 2016). Noise has even been linked as an obstacle to nurses providing care due to its prominent place as an environmental stressor (Gurses and Carayon, 2009). The quality of the healthcare experience is often evaluated by the hospital's dynamic environment (Mazer, 2016). Noise has been shown to be one of the most invasive aspects of the hospital environment (Joseph, 2006). For example, sounds of suffering patients and trauma are particularly disturbing (Mazer, 2016). In fact, patient complaints centered on noise two times more often than about anything else in the hospital, including the food (Fick and Vance, 2006). Interestingly, the opposite is an important factor in patient stress. Silence can be just as stressful for patients. When the hospital environment is "painfully silent," it "amplifies even the softest sounds" (Mazer, 2016, p. 5). As Mazer (2016) sums up nicely, the "accumulation of noise ... adds up to stress, anxiety, and in total, an unacceptable, unsatisfying, and risk-laden health experience" (p. 3).

Waiting rooms hold some of the greatest anxiety for patients. For example, sitting in the waiting room for several hours patients are exposed to the waiting room's soundscape. This may include alarms, ringing phones, people entering and exiting, or any manner of noises the room holds. As mentioned above, the sounds of other patients' experiencing trauma can play a major role in one's experience of stress as influenced by the sounds of the waiting room. Another stressor reported by patients has been the conversations overheard (or even eavesdropped) which involve other patients revealing sensitive or confidential information with another person or a healthcare provider (Mazer, 2016). The cumulative effect of these noises is often experienced as stress. Our bodies respond to noise in the same way our bodies respond to stress (Choiniere, 2010). Over time these effects impair our health through the cumulative effects of these triggers.

During my visit to several hospital sites for this study I began to notice how much my brain would become attuned to beeps, alarms, and ringing phones. The longer I sat in each space the more my mind began to hear other people. Cell phone notifications, cell phone conversations, and even interpersonal conversations moved themselves to the fore of my soundscape. Still more was the sound of people, essentially, sounding sick. Coughing, sneezing, sniveling, were very common. In the age of COVID19 they were even scary. I began to question my own position and if it was even safe to be doing research in such an environment. In one field note taking session a woman was weeping in the waiting room. I could overhear several of her phone conversations. She was not "sick" but her husband had taken a turn for the worse. After several minutes of this I realized it was having a stressful effect on me as well. After some time, her pastor came to the waiting room and they knelt on the floor and began to pray. This took place in the small midwestern town of Richmond, Indiana. To be sure, not all individuals who identify with the same faith share the same ideas and practices of that faith. Similarly, they do not share the same political viewpoint. Yet, I began to wonder how this sound might be affecting others in the waiting room. For example, what about patients who do not share the same values or patients who do not hold religious beliefs. Could someone of another religion do the same without fear of retaliation? Could this be something someone with different beliefs does not have access to because of the communication ecology in which they are currently seeking treatment? The reason this experience is so meaningful to the 
study of communication ecology and health equity is that, if any of these questions are possibilities, which I am inclined to believe they are, we see how directly impactful the space, ecology, and health equity actually are.

\section{Lighting}

A quick compare and contrast of these two approaches to waiting spaces reveals rather quickly which spaces give a nod to the patient's engagement with space and which one is more aligned with functionality. There are design elements at work here which are attuned to patient experience. For example, the carpet helps to reduce noise. In Figure 3 we notice the dropped ceiling and fluorescent light. This is intensified by the reflective back wall. The chairs offer more comfort than traditional waiting chairs with no arm rests. These are more like lounge chairs. The back wall has a pleasant design. The key in each of these examples is to notice the lighting but to also consider what values drive the design choice.

Figure 3 is a hospital waiting area in Indianapolis, Indiana. This area of town should be considered a high resourced area. According to citydata.com the average household income of this area is $\$ 109,858$ with median resident age, 39.5 (City-data.com, 2017). This data is important as it underscores the issue of space and patient well-being. Lighting choices are frequently influenced by their cost, but also by their maintenance. For example, easy maintenance often equals lower cost. Figure 5 presents another of Parsley Health's lounges offering more natural lighting combined with more soothing materials and colors. Again, the actual lighting in space is key. However, when comparing and contrasting how the light effects the space, keep in mind the values which allow that effect to manifest. Even when clinics have high resources the connection between space and patient health, care, stress, etc., is still not made.

Research demonstrates that light has a direct influence on patient stress (Sternberg, 2010). Too much light or too little light effects patient stress and even healing (Sternberg, 2010). In fact, the color of light, specifically blue light, has been shown to aid in post-stress recovery (Minguillon et al., 2017). Light has also been shown to effect comfort (Küller and Wetterberg, 1993) as well as brain activity, cardiovascular activity and even emotional states (Sroykham, 2015). Stone (1992) demonstrated that poor lighting can cause headaches and patient discomfort. Stone (1992) also added fluorescent lighting complicated by the absence of windows and lack of visual variety in interior design exerted greater influence on patient complaints than simply lighting alone. The intensity and duration of light exposure significantly effects alertness and heart rate eliciting acute physiological and alerting responses in people (Cajochen et al., 2005).

These effects on the brain and cardiovascular system influence patient comfort and stress levels. Thus, lighting should be taken seriously as part of the ecology which impacts patient wellbeing. The effect of different types of lighting is important. Yet, the values which drive the design, or said differently, which manifest themselves systemically in lighting are essential to forefronting how communication ecologies influence patient stress and health care.

\section{CONCLUSION}

Taking a more quantum view of health, communication and health equality goes beyond simple access to care. While access to care is and should be prioritized, health care and the communication ecology of the waiting room can and does create avoidable, sometimes unfair, and remediable differences among groups of people. The expression of the physical space is systemic. We see the emergence of systemic effects when we look at design choices amongst varying resource levels. In lower resourced areas these design choices are constrained by budget, income of the patients, insurance coverage, and myriad other factors. For example, a free emergency clinic may not have the resources to create spaces like we see at Parsley Health.

Yet, the purpose of the paper is not to slight those spaces which do not offer a Zen-like feeling or demand and entire rearrangement of the hospitals in America. As discussed, multiple aspects of waiting room design are part of the overall communication ecology that often perpetuates health inequities instead of helping to mitigate them. To be sure, my purpose here is not to be critical of how waiting rooms have been designed or used, especially in low resource situations. The point is to forefront waiting rooms as an under-examined systemic component patient health. It is important for any clinic to think about these issues, and as importantly, attend to them in the best way possible within their resources.

Many clinics and waiting spaces are designed for function with little attention paid to the ways in which design contributes to better or worse health, specifically patient stress responses throughout the pathways of care. Once the importance of design makes its way into the consciousness of patient care, this (in) attention becomes especially important for dealing with health inequities. Most notably, people who live in low resource communities or countries quite often get a functional design. These are intended to provide a space for care but come with no intention to mitigate patient stress responses or to help people heal. As demonstrated by Parrish-Sprowl and Parrish-Sprowl (2020) small, strategic changes to the communication ecology can make a difference as meaningful changes in the ecology of the waiting room do not require large expensive redesign projects.

The point is to show that the ecology of the space matters. It affects us. These effects are administered around social, economic, and demographical stratifications as per the WHO's definition of health equality. The space itself influences patients and healthcare workers alike at the social, emotional, and even cellular level. With this in mind, we can begin to see communication as constitutive; that is, as a process of creation. We might ask ourselves what types of interactions or relationships we are trying to create with this space instead of the waiting area simply being a holding cell for the infirmed medical object until the object has been administered some type of treatment and released. This attention to the quantum aspect of the waiting room and communication itself reorients us more advantageously to address the WHO's call for helping people attain their full health potential. 


\section{DATA AVAILABILITY STATEMENT}

The raw data supporting the conclusion of this article will be made available by the authors, without undue reservation.

\section{REFERENCES}

Ahlin, T., and Fangfang, L. (2019). From Field Sites to Field Events: Creating the Field with Information and Communication Technologies (ICTs). Med. Anthropol. Theor. 6 (2), 1-24. doi:10.17157/mat.6.2.655

Akansel, N., and Kaymakçi, Ş. (2008). Effects of Intensive Care Unit Noise on Patients: a Study on Coronary Artery Bypass Graft Surgery Patients. J. Clin. Nurs. 17 (12), 1581-1590. doi:10.1111/j.1365-2702.2007.02144.x

Azzazy, S., Ghaffarianhoseini, A., GhaffarianHoseini, A., Naismith, N., and Doborjeh, Z. (2020). A Critical Review on the Impact of Built Environment on Users' Measured Brain Activity. Architectural Sci. Rev. 64, 319-335. doi:10.1080/00038628.2020.1749980

Baldwin, A. L. (2012). How Do Plants in Hospital Waiting Rooms Reduce Patient Stress? J. Altern. Complement. Med. 18 (4), 309-310. doi:10.1089/acm.2012.0116

Braverman, P., and Gottlieb, L. (2014). The Social Determinants of Health: It's Time to Consider the Causes of the Causes. Public Health Rep. 129 (2), 19-31. doi:10.1177/00333549141291S206

Cajochen, C., Münch, M., Kobialka, S., Kräuchi, K., Steiner, R., Oelhafen, P., et al. (2005). High Sensitivity of Human Melatonin, Alertness, Thermoregulation, and Heart Rate to Short Wavelength Light. J. Clin. Endocrinol. Metab. 90, 1311-1316. doi:10.1210/jc.2004-0957

Choiniere, D. B. (2010). The Effects of Hospital Noise. Nurs. Adm. Q. 34 (4), 327-333. doi:10.1097/naq.0b013e3181f563db

City-data.com (2017). Carmel, Indiana. Retrieved From: http://www.city-data. com/city/Carmel-Indiana.html.

Coburn, A., Vartanian, O., and Chatterjee, A. (2017). Buildings, Beauty, and the Brain: A Neuroscience of Architectural Experience. J. Cogn. Neurosci. 29 (9), 1521-1531. doi:10.1162/jocn_a_01146

Cohn, S., Clinch, M., Bunn, C., and Stronge, P. (2013). Entangled Complexity: Why Complex Interventions Are Just Not Complicated Enough. J. Health Serv. Res. Pol. 18 (1), 40-43. doi:10.1258/jhsrp.2012.012036

Cozolino, L. (2014). The Neuroscience of Human Relationships. New York: W. W. Norton

Cunha, M., and Silva, N. (2015). Hospital Noise and Patients' Wellbeing. Proced. Soc. Behav. Sci. 171, 246-251. doi:10.1016/j.sbspro.2015.01.117

Dunckley, V. L. (2014). Gray Matters: Too Much Screen Time Damages the Brain: Neuroimaging Research Shows Excessive Screen Time Damages the Brain. Psychol. Today (Accessed July 7, 2020).

Endevelt-Shapira, Y., Perl, O., Ravia, A., Amir, D., Eisen, A., Bezalel, V., et al. (2017). Altered Responses to Social Chemosignals in Autism Spectrum Disorder. Nat. Neurosci. 21, 111-119. doi:10.1038/s41593-017-0024-x

Fick, D. D., and Vance, G. L. (2006). Quiet Zone. Reducing HVAC System Noise. Health Facil. Manage. 19 (8), 21-24.

Grosser, B. I., Monti-Bloch, L., Jennings-White, C., and Berliner, D. L. (2000). Behavioral and Electrophysiological Effects of Androstadienone, a Human Pheromone. Psychoneuroendocrinology 25, 289-299. doi:10.1016/s0306-4530(99)00056-6

Gurses, A. P., and Carayon, P. (2009). Exploring Performance Obstacles of Intensive Care Nurses. Appl. Ergon. 40 (3), 509-518. doi:10.1016/j.apergo.2008.09.003

Hannerz, U. (2003). Being there. And there. and There!. Ethnography 4 (2), 201-216. doi:10.1177/14661381030042003

Hannigan, T. P. (1995). Body Odor: The International Student and Cross-Cultural Communication. Cult. Psychol. 1 (4), 497-503. doi:10.1177/1354067x9514006

Hine, C. (2000). Virtual Ethnography. London: SAGE.

Hood, K. K., Beavers, D. P., Yi-Frazier, J., Bell, R., Dabelea, D., Mckeown, R. E., et al. (2014). Psychosocial burden and Glycemic Control during the First 6 Years of Diabetes: Results from the SEARCH for Diabetes in Youth Study. J. Adolesc. Health 55 (4), 498-504. doi:10.1016/j.jadohealth.2014.03.011

Hullinger, J. (2020). 12 Ways Airport Are Secretly Manipulating You. New York: Mental Floss. Retrieved from: https://www.mentalfloss.com/article/64808/12behind-scenes-secrets-airports (Accessed July 7, 2020).

\section{AUTHOR CONTRIBUTIONS}

The author confirms being the sole contributor of this work and has approved it for publication.

Joseph, A. (2006). The Role of the Physical Environment in Promoting Health, Safety, and Effectiveness in the Healthcare Workplace. Concord, CA: The Center for Health Design. Issue Paper \#3.

Küller, R., and Wetterberg, L. (1993). Melatonin, Cortisol, EEG, ECG and Subjective comfort in Healthy Humans: Impact of Two Fluorescent Lamp Types at Two Light Intensities. Lighting Res. Techn. 25, 71-80. doi:10.1177/ 096032719302500203

Ladd, K. (2019). This Modern Healthcare Startup Is Using Design to Heal. Architectural Digest, New York, November 14, 2019 Trevor Tondo: Photo credit (Retrieved June 15, 2020).

Lamb, M. D. (2014). Misuse of the Monument: The Art of Parkour and the Discursive Limits of a Disciplinary Architecture. J. Urban Cult. Stud. 1 (1), 107-126. doi:10.1386/jucs.1.1.107_1

Lamb, M. D. (2017). Traceur as Bricoleur. Poaching Public Space through Bricolent Use of Architecture and the Body. Jps 2 (1), 33-44. doi:10.5204/ jps.v2il.4810.5204/jps.v2i1.48

Lamb, M. (2014). "Self and the City: Parkour, Architecture, and the Interstices of the 'Knowable' City. Liminalities: A J. Perform. Stud. 10 (2), 1-20.

Marcus, G. E. (1995). Ethnography In/of the World System: The Emergence of Multi-Sited Ethnography. Annu. Rev. Anthropol. 24, 95-117. doi:10.1146/ annurev.an.24.100195.000523

Mazer, S. E. (2016). Hospital Noise \& the Patient Experience. Retrieved http:// www.healinghealth.com/wp/wp-content/uploads/2014/08/Mazer_HospitalNoise-and-The-Patient-Experience.pdf(Accessed June 6, 2016).

Minguillon, J., Lopez-Gordo, M. A., Renedo-Criado, D. A., Sanchez-Carrion, M. J., and Pelayo, F. (2017). Blue Lighting Accelerates post-stress Relaxation: Results of a Preliminary Study. Plos One 12, e0186399. doi:10.1371/ journal.pone.0186399

Molhave, L. (1989). The Sick Buildings and Other Buildings with Indoor Climate Problems. Environ. Int. 15 (1-6), 65-74.

Parrish-Sprowl, J., and Parrish-Sprowl, S. (2017). Communication for Whole Health. Indianapolis, Indiana: GHCC.

Parrish-Sprowl, J., and Parrish-Sprowl, S. (2014). "Transforming Trauma: Space for Growth and Meaning-Making after Adversity," in Conference paper, International Transformative Learning Conference, October, 2014 (Columbia University). doi:10.13140/2.1.2372.3208

Parrish-Sprowl, J., Parrish-Sprowl, S., and Alajlouni, S. (2020). Innovations in Addressing Mental Health Needs in Humanitarian Settings: A Complexity Informed Action Research Case Study. Front. Commun. Health Commun. doi:10.3389/fcomm.2020.601792

Pink, S. (2015). Digital Ethnography: Principles and Practice. London: Sage.

Postman, N. (2000). The Humanism of media Ecology. Proc. Media Ecol. Assoc. Vol.1, $10-16$.

Ramachandran, V. S. (2011). The Tell-Tale Brain: A Neuroscientist's Quest for what Makes Us Human. New York: W. W. Norton.

Rutter, W. M. (2018). Using Flawed, Uncertain, Proximate and Sparse (FUPS) Data in the Context of Complexity: Learning from the Case of Child Mental Health. BMC Med. 16, 82. doi:10.1186/s12916-018-1079-6

Spinella, M. (2009). A Relationship between Smell Identification and Empathy. Int. J. Neurosci. 112 (6), 605-612. doi:10.1080/00207450290025680

Sroykham, W. (2015). The Red and Blue Rooms Affect to Brain Activity, Cardiovascular Activity, Emotion and Saliva Hormone in Women," in BMEiCON 2014-7th Biomed Eng Int Conf. 2015, Fukuoka, January, 22, 2015 (Geneva).

Sternberg, E. M. (2010). Healing Spaces: The Science of Place and Well-Being. Boston: Harvard Univ Press.

Stone, P. T. (1992). Review Paper: Fluorescent Lighting and Health. Lighting Res. Techn. 24 (2), 55-61. doi:10.1177/096032719202400201

Sullivan, R. M., Wilson, D. A., Ravel, N., and Mouly, A.-M. (2015). Olfactory Memory Networks: From Emotional Learning to Social Behaviors. Front. Behav. Neurosci. 9, 36. doi:10.3389/fnbeh.2015.00036 
Tamas, R., Ouf, M. M., and O Brien, W. (2020). A Field Study of the Effect of Building Automation on Perceived comfort and Control in Institutional Buildings. Architectural Rev. 63 (1), 74-86.

T. Brunyé, T., R. Mahoney, C., L. Gardony, A., and A. Taylor, H. (2010). North Is up(hill): Route Planning Heuristics in Real-World Environments. Mem. Cogn. 38 (6), 700-712. doi:10.3758/mc.38.6.700

World Health Organization Who (2020). Health Equity Geneva. (Accessed July 4,20$)$.

Conflict of Interest: The author declares that the research was conducted in the absence of any commercial or financial relationships that could be construed as a potential conflict of interest.
Publisher's Note: All claims expressed in this article are solely those of the authors and do not necessarily represent those of their affiliated organizations, or those of the publisher, the editors and the reviewers. Any product that may be evaluated in this article, or claim that may be made by its manufacturer, is not guaranteed or endorsed by the publisher.

Copyright $\odot 2021$ Lamb. This is an open-access article distributed under the terms of the Creative Commons Attribution License (CC BY). The use, distribution or reproduction in other forums is permitted, provided the original author(s) and the copyright owner(s) are credited and that the original publication in this journal is cited, in accordance with accepted academic practice. No use, distribution or reproduction is permitted which does not comply with these terms. 